\title{
Protée
}

\section{Lumières et profondeur épistémique}

\section{Françoise Parouty-David}

Volume 31, numéro 3, hiver 2003

\section{Lumières}

URI : https://id.erudit.org/iderudit/008440ar

DOI : https://doi.org/10.7202/008440ar

Aller au sommaire du numéro

Éditeur(s)

Département des arts et lettres - Université du Québec à Chicoutimi

ISSN

0300-3523 (imprimé)

1708-2307 (numérique)

Découvrir la revue

Citer cet article

Parouty-David, F. (2003). Lumières et profondeur épistémique. Protée, 31(3), 91-99. https://doi.org/10.7202/008440ar

\section{Résumé de l'article}

Parti d'un énoncé pictural de nature religieuse de par son titre, La Mort de la Vierge du Caravage, l'article s'efforce de montrer la capacité de la lumière et de l'ombre à modaliser des repères qui en modifient l'appartenance catégorielle. Il s'appuie sur des opérations propres à la synesthésie pour décrire, dans un premier temps, comment la lumière, qui régit principalement le visible, donne accès à des sensations auditives et kinesthésiques. Elle a donc une fonction homogénéisante, d'un point de vue sémantique, qui souligne le rôle du corps dans la saisie de l'oeuvre d'art. Au-delà, les variations de son intensité sont mises en rapport avec les capacités cognitives du sujet percevant, amené à explorer une plus grande profondeur. 


\title{
LU M IÈRES ET PRO FO N DEU R ÉPISTÉM IQ U E
}

\author{
FRANÇOISE PAROUTY-D AVID
}

[...] voir c'est toujours voir plus qu'on ne voit.

Merleau-Ponty (2002: 295)

La sculpture ou l'architecture produisent des objets plastiques phénoménologiquement déployés dans un espace réel et appréhendés dans cet espace en trois dimensions, qui intègre les mouvements de la lumière naturelle. Il en est autrement de l'artefact pictural:

Quoi qu'on puisse dire par ailleurs de la lumière, on ne peut nier qu'elle soit d'une légèreté absolue, ni lourde ni résistante; au contraire elle est purement identique à elle-même et ne se rapporte qu'à elle-même; c'est la première idéalité, la première identité dans la nature. Dans la lumière, la nature commence à devenir subjective [...]. Par cette qualité plus idéale, la lumière devient le principe physique de la peinture. (Hegel, 2002: 22)

Tel est l'un des principes fondamentaux de la leçon d'esthétique de Hegel. La peinture réduit ces trois dimensions à la surface plane, elles ne sont donc pas "présentes en elles-mêmes, dans leur réalité propre; elles ne sont rendues apparentes et visibles que par cet élément physique» (ibid.: 24), par la représentation de la lumière et de son ombre, du clair et de l'obscur. En regard de ce préalable théorique, on peut considérer celui, pragmatique, d'Henri Alekan, chef-opérateur de cinéma et manipulateur de lumières: «L'apparence des choses, des formes, est le fait de la lumière. Son absence détruit l'objet, puisqu'il n'est plus perçu. [...] La lumière donne à voir, mais plus encore, elle donne à penser» (2001: 12-14). D'un point de vue sémiotique enfin, toute spéculation sur la peinture apparaît comme une spéculation sur la lumière. La configuration sémiotique de la lumière se présente comme

[...] une construction dont les catégories constitutives devraient permettre de décrire les effets de sens nés des interactions (déictiques, modales, passionnelles...) entre l'activité perceptive-énonciative d'un sujet et le gradient de l'énergie. (Fontanille, 1995: 28) 
L'histoire de l'art offre, dans sa classification, quelques repères aisés sous des dénominations historiques qui figurent cette dialectique de la lumière en peinture: clair-obscur, luminisme, ténébrisme, nocturnes, diurnes, etc., et c'est dans ce corpus que nous puiserons une œuvre particulière du Caravage ${ }^{1}$ visible au Louvre: La Mort de la Vierge (1606). La scène illustre un très bref récit biblique, qui a donné lieu à maintes représentations peintes ou sculptées, dans lequel se retrouvent toujours les mêmes acteurs: la Vierge entourée des douze apôtres, de MarieMadeleine et parfois du Christ. La mort de la Vierge est habituellement considérée comme un court sommeil d'où elle fut miraculeusement enlevée par les anges pour être conduite au $\mathrm{Ciel}^{2}$. Notre critère de choix est l'intérêt de la coprésence de deux registres distincts dans un énoncé qui relève simultanément de l'humain et du divin: du divin par son titre, la référence mythique qu'il implique ainsi que l'auréole de Marie, bien qu'à peine visible; notre perception est alors guidée par un savoir plaqué sur l'énoncé plus que par une réelle capacité à voir, qui cependant nous conduirait à discrétiser d'autres repères de l'ordre de l'humain. Notre première tâche sera d'évaluer dans quelle mesure la lumière intervient dans cette possible appartenance à l'une ou l'autre de ces catégories. Elle nous conduira à analyser comment la lumière participe de la composition d'ensemble pour construire une synesthésie, puisqu'elle ne donne pas seulement à voir, mais aussi à entendre et à toucher, en soulignant le rôle du corps dans la saisie de l'œuvre d'art. Au-delà, nous valoriserons l'interaction entre cet objet particulier, animé des variations de l'intensité lumineuse, et le sujet percevant, qui est amené à explorer une plus grande profondeur épistémique.

\section{OÚ L'ART DE FAIRE APPARAÎTRE DEVIENT LE PRINCIPAL OBJET}

Évidence: la lumière fait voir. Mais quoi et comment? Condition de visibilité, la lumière est bien le principe physique de toute représentation picturale, et les propriétés de la lumière procèdent de la composition d'ensemble de toute image, puisque la

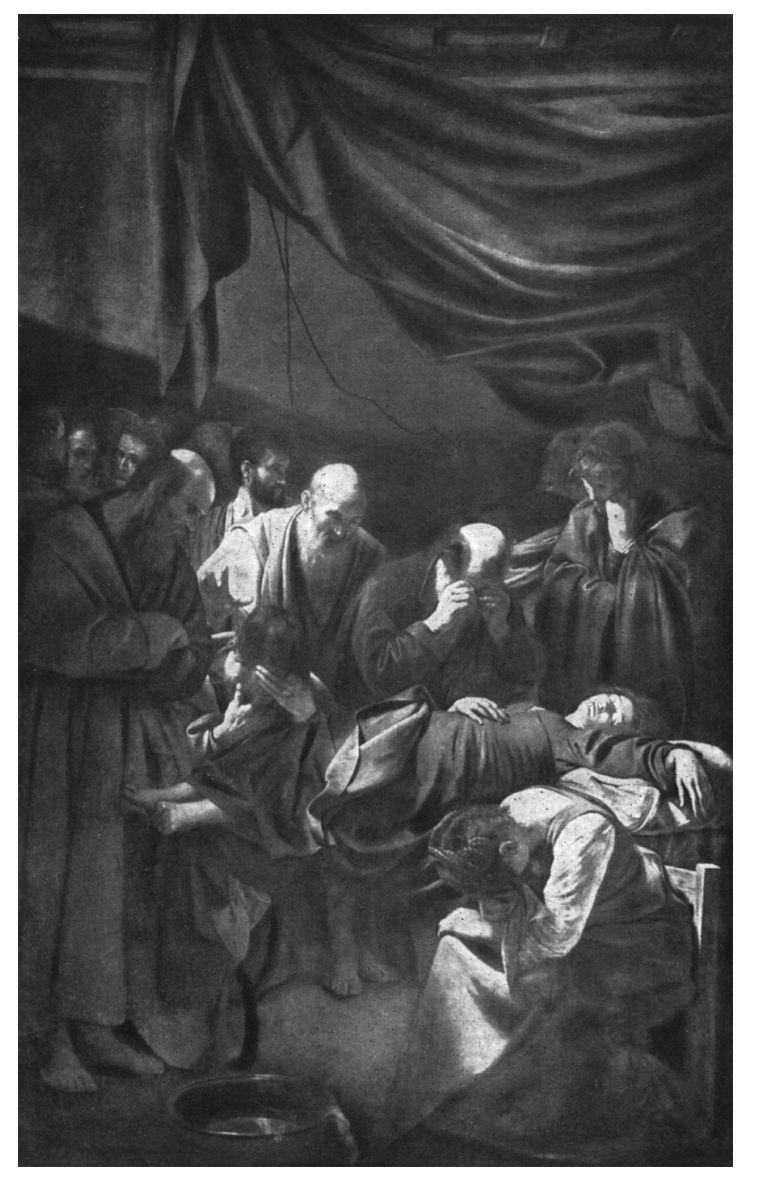

Le Caravage, La Mort de la Vierge, 1606. 2,45 x 3,69 m. Musée du Louvre, Paris.

perception est sémiotisée. Ces propriétés construisent les lignes structurantes de l'expression et du contenu, étant donné qu'elles sont un élément de la sémiose: topologique, puisqu'elle conjoint ou disjoint des points de l'espace énoncé et, en cela, le hiérarchise; éidétique, puisqu'elle révèle ou oblitère les contours et les volumes; chromatique, puisque la couleur est un phénomène physique, un produit de la lumière. Elle indique de la sorte un parcours à effectuer dans l'espace énoncé en fonction des oppositions de toute nature, plus ou moins fortement accentuées.

La Mort de la Vierge offre une perspective peu profonde, réaliste, dans une scène d'intérieur dont on aperçoit le plafond et le mur du fond. Dans cet arrière-plan, les sources de lumière hors champ déterminent des zones ombrées, qui dessinent la verticalité du mur par un léger modelé, et participent ainsi de la mise en place de cette faible profondeur, tandis que des zones plus lumineuses avancent vers 
l'observateur, quelque peu segmentées et orientées par des points d'impact sur les acteurs. Si l'on retient l'idée d'une échelle de clarté, comme celle qu'emploient les opérateurs de cinéma pour rendre compte des gradients de lumière présents dans l'image et qu'ils objectivent avec une cellule photoélectrique, on peut dire que l'énoncé présente une échelle très courte d'un noir transparent à l'ocre clair sans blanc, en passant par le rouge. Dans cet ensemble de faible amplitude, la saisie reste impressive et la qualité de la lumière, qui se laisse mal cerner, évoque une luminosité diurne qui perce à l'économie dans un espace restreint. Lorsque nous parlons de lumière dans un énoncé pictural, rappelons qu'il s'agit de sa représentation chromatique, de la plasticité des procédures qui en donnent l'illusion et par lesquelles, il faut bien le reconnaître, elle éclaire ou assombrit l'ensemble. On sait par ailleurs, et Alekan le signale, que, sans effet solaire, la perception du temps, comme moment de la journée, disparaît d'une image: à l'aurore correspondent des lignes de lumière horizontales, au zénith, des lignes verticales et, entre les deux, des obliques, qui situent dans des temps intermédiaires précisés par l'angularité des ombres projetées. Chez Le Caravage, la vectorialisation oblique de l'espace, par la percée lumineuse dans une obscurité dominante, temporalise la scène, mais de manière imprécise: début ou fin de journée, peu importe, puisque, dans l'illustration d'un récit biblique, l'essentiel est d'imposer une équation entre lumière et sacré. À cette ambiguïté s'ajoute l'effet de clôture, produit tout autant par l'ombre dominante que par le cadrage, qui tire encore l'énoncé vers une temporalité incertaine et figée; cet effet de clôture a pour conséquence de stopper le programme narratif dans un temps qui se dilate, dans une sorte de déhiscence du temps. En somme, ces opérations construisent un univers baroque type, au sens où l'entend Deleuze:

[...] la chambre obscure n'a qu'une petite ouverture en haut [...] la lumière ne pénètre que par des orifices si bien coudés qu'ils ne laissent rien voir $d u$ dehors, mais illuminent ou colorent les décorations d'un pur dedans. (1997: 40)
Et l'on imagine aisément la seconde enveloppe isomorphe que serait celle de l'édifice religieux, auquel le tableau était destiné, et qui accentuerait encore l'effet de clôture. La saisie ne peut qu'en être ralentie et le tableau constitue ce que Merleau-Ponty appelle un monde, c'est-à-dire:

[...] un ensemble organisé, qui est clos, mais qui étrangement est représentatif de tout le reste, possède ses symboles, ses équivalents pour tout ce qui n'est pas lui. La peinture pour l'espace par exemple. (2002: 272)

Il nous semble que c'est tout aussi vrai de la lumière qui définit l'espace représenté. Le tableau n'est donc pas ouverture sur un espace, mais embrayage, jonction par pénétration et prolongement du champ de l'observateur dans ce monde clos.

Les mêmes éléments sensibles y signifient autre chose que dans

le monde prosaïque [...], la structure sensible ne peut être

comprise que par sa relation au corps, à la chair. (Ibid.: 273)

C'est pourquoi nous soulignerons ultérieurement la nécessaire fusion des sens dans la synesthésie comme source $d u$ sens.

À ce stade de l'observation, nous pouvons hésiter entre deux formulations qui ont des conséquences sémantiques très différentes. C'est là un point crucial qui oriente le sens global de l'énoncé et qui correspond à une intentionnalité lisible dans les modalités de manipulation discursive menant à des fins rhétoriques: soit nous dirons que les objets éclairés orientent l'attention de l'observateur sur la ligne de lumière qui, de proche en proche, les conjoint, et à laquelle ils renvoient de manière indicielle; soit que, inversement, le parcours de la lumière attire le regard sur les objets qu'elle révèle. - Dans le premier cas, la lumière se fait voir. Le rideau, qui théâtralise la scène en formant comme un grand dais au-dessus du tiers de la toile, et dont la chute verticale est la partie la plus éclairée, donne le ton, au sens propre d'un énoncé où le rouge domine. La réflexion lumineuse qui détermine la saturation de la couleur a une fonction indicielle, elle renvoie le regard vers la source et la désigne: la lumière est à 
elle-même sa propre cible par réflexion. Le vêtement de la Vierge, de même couleur, a la même fonction, mais cette fois accentuée, parce qu'il est cerné des seules taches claires de l'ensemble qui créent un embrayage sensible plus rapide. La lumière, hors champ et seulement réfléchie, devient la cible finale de l'observateur dans l'espace d'énonciation, elle régit le regard du bas vers la périphérie haute et extérieure au format vertical: «s'extravasant», elle tire l'interprétation vers le divin, selon un des traits de l'inventaire baroque chez Wölfflin, et demeure cohérente avec le titre du tableau qui joue le rôle d'attracteur sémantique.

- Dans la seconde hypothèse, qui n'invalide pas la précédente, les fonctions sont inversées. La lumière donne à voir, elle est agent, sa fonction est monstrative et concerne tous nos sens: le regard s'arrête dans son parcours, les corps sont l'ultime cible et, avec eux, l'humain dans sa chair. Le rayon lumineux oblique éclaire des parties pour construire un tout signifiant. Métonymiques des corps laissés dans l'ombre, ces parties ont pour justification d'élaborer l'isotopie de la douleur qui imprime la chair. En somme, le rai de lumière arbitre les formes, opère une déclinaison lumineuse des parties du corps où nous pouvons lire la pathémisation. Le rai synthétise à lui seul le schéma narratif fondé sur l'opposition mort/vie conjuguée à l'opposition repos/souffrance. Toute une ontologie parcourt ce trait de lumière dominant.

Dans ce tableau commenté par Alekan, le schéma de la lumière le confirme. Alekan le construit sur une discrétisation, à la fois qualitative et quantitative, entre lumières directionnelles principales, secondaires et tertiaires. Les premières, écrit-il, constituent

[l']axe principal [qui] devient le siège du développement

de toute la structure-lumière qui va s'organiser antour et avec lui [...]. Il domine l'ensemble par sa directivité et la puissance de son flux: c'est la lumière-clef. (Alekan, 2001: 127)

Les secondaires architecturent «le lieu par un volumelumière» et sont d'une puissance inférieure. La lumière tertiaire est «due aux multiples jeux des surfaces à matériaux réfléchissants. C'est par excellence la lumière des nuances et des transitions" (ibid.). Le schéma atteste de la primauté absolue de la direction oblique et descendante décrite dans la mise en place des lumières, tertiaires pour la plus large part. Toutes convergent sur le corps mort et sur les plus proches acteurs. C'est presque une lumière unidirectionnelle à laquelle Alekan accorde un pouvoir-hiérarchiser:

[...] en éclairage unidirectionnel l'intensité du flux lumineux, engendrant les jeux d'ombres, crée de ce fait des rapports intensité-densité proportionnels au flux lumineux qui a été générateur du complexe lumière-ombre: plus l'intensité lumineuse est grande et plus la densité des ombres est accusée, et inversement. (Ibid.: 34)

À la périphérie proche de cette diagonale dominante, le conflit entre ombre et lumière se résout dans son terme complexe, le clair-obscur, là où la lumière est plus diffuse et pourvue d'ombres qui pathémisent encore davantage l'énoncé en affirmant la richesse des sensibilités contenues et maintenues dans l'ombre. En fait, les couleurs jaillissent du noir et la technique de préparation, présentée par F. Bardon, l'explique:

Le travail consiste à affronter la surface du support [...] cette surface n'est pas nulle ni ne sert de repoussoir aux couleurs, mais la préparation à base de terre d'ombre est une couleur avec laquelle les autres couleurs doivent compter, l'ocre et le rouge sont posés dans ce noir, et l'acte de peindre se situe entre le noir et la forme/couleur, laquelle émerge dans son fond originel qu'elle modifie autant qu'elle est conditionnée par lui.

(Bardon, 1997: 150) 3

Les rouges assurent «le triomphe du noir, comme matière, matrice originelle de toute chose» et sont comme «suspendus dans cette noirceur souterraine» aux modulations savantes «noir bruni, grisé, beigé, ocré, rougeoyant, bleuissant» (ibid.: 78). Greimas corrobore cette figure chromatique de la rétention quand il note, dans De l'imperfection:

L'absence de couleur qu'est le noir cache donc une présence bariolée explosive. Les ténèbres parfaites contiennent virtuellement toutes les couleurs, toute la beauté du monde, elles sont la couleur protopathique. (Greimas, 1987: 51) 
Dans les œuvres ultérieures du Caravage (David tenant la tête de Goliath), on sait que la syntaxe chromatique «nu ocre/drapé rouge/fond noir» évoluera vers la disparition du rouge pour qu'éclate le seul conflit ombre/lumière dans un contraste maximal. Il est certain que, d'un point de vue anthropologique, le noir, perçu comme générateur d'angoisse, est dysphorique. Cette conception est fondée sur de multiples explications: notre néant originel, l'anéantissement de la perception visuelle et de l'action, la convocation de sens substitutifs moins exercés, tout se conjugue pour accentuer le ralentissement dans la saisie cognitive et augmenter la pathémisation.

Le conflit entre l'effet d'éclairage et l'effet de chromatisme est particulièrement perceptible à l'extrémité de la diagonale. Ainsi, quand le rai de lumière oblique se heurte à l'horizontale rouge et lumineuse du corps mort qui obstrue cette verticalité du format dans un espace plein et sombre, il aboutit, comme toutes les autres lignes, sur cet actant de contrôle opaque qui concentre l'intensité lumineuse, obstacle tangible et dense de la mort du corps, soit au somatique et non au divin. Paradoxalement, par ce choc visuel, l'observateur est conduit à l'invisible et à l'irreprésentable, au spéculatif sur la grande question, celle de la mort, univers obscur où le sens s'approche difficilement dans les modulations du rouge jailli du noir.

D'où le procédé de l'ébauche, qui est le procédé supérieur de la technique de l'émergence. L'ébauche ne concerne pas le seul détail, tel le pied de la Vierge, à peine sorti. Elle est un véritable moyen d'expression, une manière de connaissance du réel qui ne se laisse cerner que par fragments. (Ibid.: 191)

Nous comprenons ce rapport visible/invisible à la manière de Merleau-Ponty, qui recommande dans ses notes de travail:

[de] ne pas considérer l'invisible comme un autre visible "possible», ou un "possible» visible pour un autre: ce serait détruire la membrure qui nous joint à lui. [...] L'invisible est là sans être objet, c'est la transcendance pure, sans masque ontique. Et les «visibles» eux-mêmes, en fin de compte, ne sont que centrés sur un noyau d'absence eux aussi. (2002: 278)
C'est dans ces modalités du visible qui ouvrent à l'invisible, et qui sont régies par la lumière, que se joue la synesthésie.

\section{OMBRE ET SILENCE}

Le Caravage éclaire avec parcimonie, d'une part, les crânes chauves, le visage de la Vierge de troisquarts-face et la nuque découverte de la pleureuse prostrée et, d'autre part, essentiellement la chair des mains des apôtres lorsqu'elles surgissent de l'ombre. C'est aux mains éclairées que nous lirons d'abord le mutisme. De gauche à droite, la première main ouverte aux doigts tendus marque l'arrêt lié à la surprise; la deuxième, sur une bouche, retient un cri; une autre essuie des larmes; une quatrième, contractée, supporte un visage méditatif; la dernière prend appui sur un genou pour soutenir le poids du corps. Toutes, dans les ocres plus ou moins saturées de la chair, sont crispées, dans la rétention, et seules les deux mains de la Vierge sont inertes. Dans La Mort de la Vierge, pas de traits spécifiques aux visages représentés, seuls comptent mains et crânes soumis à la lumière des vérités passionnelles profondes, incarnées et muettes, dans des êtres qui comptent plus pour ce qu'ils figurent que pour leur «apparaître».

La déformation des quatre bouches ouvertes le confirme. Elles peuvent laisser supposer gémissements et lamentations, parce qu'elles sont dans une obscurité qui étouffe les bruits, ce qui contribue à l'idée que, plus que la parole articulée, elles sont l'expression d'une sensation. La forme amplifiée de telles manifestations, comme le cri, éclaterait nécessairement en pleine lumière parce qu'il y a une concordance des intensités dans les signes de la représentation. Le silence est lié à la valeur pathémique de l'ombre dans une narration; ils sont du même ordre, celui de l'obstruction qui crée un obstacle dans l'accès au visible comme à l'intelligible. C'est dans ce sens que l'entend Fontanille dans sa modalisation cognitive de l'espace: «L'obstruction caractérise tout ce qui est masqué, difficilement saisissable, incomplet, ou peu reconnaissable, comme négation de l'exposition» (1989: 55). Cette modalité 
particulière à fonction d'atténuation rejoint la thèse de N. Roelens ${ }^{4}$, selon laquelle le cri perçu dans la bouche ouverte «enlève à l'humain son attribut constitutif: le verbe comme propre de l'homme». Nous considérons que le cri est, en effet, «une action bestiale, inhumaine", mais que la retenue qui caractérise notre énoncé recatégorise en humain du fait de la rection des forces d'expression. Il n'en demeure pas moins que la mimique buccale est de l'ordre du gestuel et de l'expression de l'affect. En somme, la douleur ne se dit pas ou se dit à voix basse, mais la lumière toujours réduite la souligne dans une gestuelle tout en retenue. De même qu'il faut du temps pour saisir les détails dans l'ombre, il faut du temps pour pénétrer ce monde de sensibilité tacite: les variations de l'intensité lumineuse impliquent une accommodation.

Retenue des gestes dans la lumière et extériorisation modérée des cris étouffés par l'ombre: tout se passe dans le ténu, comme si l'intensité lumineuse actantialisait les individus, la clarté incitant à la rétention du geste, l'ombre favorisant un peu l'extériorisation par la voix. Tout se passe aussi comme si s'exerçait une complémentarité, entre signes iconiques et plastiques, en forme de chiasme, complémentarité entre deux faces d'un tout explicité par l'énoncé visuel du Caravage qui met en scène les perceptions en connivence. Merleau-Ponty utilise le trope:

Il faut bien que la parole entre chez l'enfant comme silence, perce jusqu'à lui à travers le silence et comme silence [...]. C'est ce négatif fécond qui est institué dans la chair, par sa déhiscence - le négatif, le néant, c'est le dédoublé, les 2 feuillets du corps, le dedans et le dehors articulés l'un sur l'autre. [...] Il n'est pas besoin d'un spectateur qui soit des 2 côtés. Il suffit que, d'un côté, je voie l'envers du gant qui s'applique sur l'endroit, que je touche l'un par l'autre [...] le chiasme est cela: la réversibilité. (2002: 211)

Il nous semble que la réversibilité est articulée ici par cette synesthésie qui nous donne à comprendre que la lumière, dans ses variations, est le vecteur qui conjoint des manifestations réparties sur plusieurs de nos sens.
Ce chiasme sensoriel entre visible et audible fait émerger le sens qu'il faut chercher dans une zone encore plus dense, plus invisible:

Le seul lieu où le négatif soit vraiment, c'est le pli, l'application

l'un à l'autre du dedans et du dehors, le point de retournement -

[...] il y a dedans et dehors tournant l'un autour de l'autre.

(Ibid.: 311-312)

\section{LA COURBURE ET LE PLI}

Certes, nous l'avons vu, l'intensité discrétise les configurations des crânes, les reliefs noueux des mains osseuses comme l'aplat de la nuque lisse de la jeune fille; elle fait toucher la chair par synesthésie qui, si elle conjoint le plus souvent les sensations et leurs représentations visuelles et auditives, recouvre également des correspondances tactiles, voire kinesthésiques. Et cette nuque attire notre attention sur toutes les lignes courbes de l'énoncé qui ne contient aucune ligne droite, si l'on excepte les vecteurs virtuels qui schématisent les tracés de lumière. Elle nous semble surtout prototypique des attitudes de tous ces corps inclinés et comme re-pliés sur eux-mêmes, dans un mouvement proprioceptif qui signe encore la retenue. Mais ce corps-là est, au premier plan, pointé par l'extrémité de la diagonale de lumière, et vu de dos dans toute l'éloquence d'une telle corporalité sur laquelle le regard se fixe, en outre parce qu'il parfait le cercle des témoins et qu'ainsi «il devient, en termes barthésiens, le punctum de la toile» (Banu, 2000: 50). Banu situe historiquement la position de dos à la première Renaissance:

$$
\begin{aligned}
& \text { Auparavant, consacrée à son dialogue avec le sacré, la peinture } \\
& \text { religieuse ignore le personnage vu de dos, car personne ne peut se } \\
& \text { dérober à la surveillance de l'œil divin et nulle dérogation n'est } \\
& \text { possible. L'Homme de dos ferait écran et brouillerait l'échange, } \\
& \text { car, par sa simple posture, il affirmerait un début } \\
& \text { d'indépendance, l'amorce d'une autonomie. (Ibid.: 47-48) }
\end{aligned}
$$

Montrer le dos s'inscrit donc en rupture avec la référence définitive au sacré et, simultanément, cristallise la rupture avec l'observateur. Caravage, par le choix de ce procédé d'énonciation, souligne la capacité de la lumière à désigner encore un repère qui 
s'ajoute aux précédents pour modifier l'appartenance catégorielle de l'énoncé: à l'apparente lisibilité canonique se substitue une interprétation de l'ordre du secret et de l'humain, dans sa dimension pathémique. Le corps vu de dos est le signe de l'ambigu, de l'hypothétique, le contraire de l'absolu, du dogmatique; soustrait à l'exposition du visage, il est le signe de l'absorption dans une intériorité où l'on s'absente du monde en s'isolant du groupe. En créant une perturbation du visible, le corps écarte l'observateur du réel et le conduit à l'invisible parce qu'il désigne un espace encore plus clos sur une obscurité totale (et un silence acquis par l'isolement), car le corps est coupé de la pleine lumière où la nuque est exposée. La position contraire serait la verticalité, la frontalité et la verbalisation qui fondent la pratique théâtrale dans la majorité des cas et qui reconnaissent l'altérité. Ce corps nous montre le chemin du dehors au dedans, là où «ce que l'on ne voit pas a la priorité sur ce qui se montre" (ibid.: 158).

Cet effet de profondeur se retrouve dans les drapés et les étoffes, dont le trait commun est le pli. Deleuze remarque combien les plis accrochent la lumière dans les effets de matière et de texture en raison de «la concavité et la convexité du rayon lumineux» (1997: 52). Saturant ou désaturant les reliefs et les creux, celui-ci accuse l'aspect, la souplesse et la lourdeur qui créent la forme, jamais donnée mais construite par la lumière. Nourri de la philosophie de Leibniz, Deleuze attribue aux matières et aux textures - toutes indifférenciées ici - le pouvoir de renvoyer à des forces primitives, qui sont celles de l'âme, et aux plis de poser la forme comme "paysage mental». Ils habillent l'ombre, désignée par la pointe du tombé rouge, où les corps sont tous également revêtus de plis qui brisent les enveloppes corporelles et noient les contours dans l'unité forte d' "une puissance d'enveloppement et de développement» (ibid.: 33). Serrés les uns contre les autres dans ce continuum d'opacité «diversifié par les plis comme un derme à vif " (ibid.: 6), ils induisent, à partir de cette synesthésie tâtonnante et dysphorique, un parcours indéterminé, subjectif et intérieur. Toutes ces têtes courbées ignorent le grand drapé au-dessus d'elles qui n'offre aucune percée vers une quelconque sphère supérieure, un autre «étage» qui, dans l'art baroque, serait représenté, selon Deleuze, au-delà du pli séparateur entre haut et bas. Sans échappée possible chez Le Caravage, le refuge se situe dans cet autre espace complexe et infini, figuré dans les multiples replis noirs de la matière. Ils sollicitent donc des forces intérieures, et les rares objets éclairés du tableau deviennent destinateurs pour l'observateur, qui est guidé vers les zones d'ombre et de fascination. «Ces plis [..., constitués sur la toile opaque, représentent les connaissances innées mais qui passent à l'acte sous les sollicitations de la matière» (ibid.).

S'il nous paraît impossible, au terme de cette analyse, de proposer une hiérarchie figée des sensations qui se confrontent dans les processus synesthésiques, il nous semble indispensable de distinguer le cas particulier des artefacts picturaux (produits pour être vus dans la majorité des cas). Dans ce cadre, le voir, composante majeure, médiatise et asservit toute autre sensation qui pourrait être dite mineure, mais il demeure lui-même régi par l'utilisation que l'énonciateur fait de la lumière. Celuici a donc la capacité de modaliser l'observateur en effectuant des manipulations plastiques qui construisent une rhétorique propre.

\section{DU DEHORS AU DEDANS}

C'est par les effets de lumière que le dehors et le dedans se rejoignent, créant un système unifié autour des rapports intimes et charnels avec les choses secrètes ou mystérieuses ou, bien entendu, métaphysiques. La faible intensité dominante est riche de conséquences: par l'homogénéisation des formes et des couleurs, elle incite l'observateur à dépasser les apparences et les surfaces pour pénétrer l'invisible par la connivence des sens et à se laisser enclore dans cet espace incommensurable et profond, ou envoûter, dirait encore Merleau-Ponty. Le vecteur de clarté conserve sa fonction monstrative et permet l'émergence de quelques points d'entrée dans cette enveloppe close sur le sombre, l'étouffé, le replié, le dense, le dedans, d'où la figure totalisante de la concentration, qui est 
convergence et contention de l'épars. Il apparaît alors que, à l'inverse de ce qui se produit dans le monde réel, les tensions sont chiasmatiques: le plus de lumière restreint la perception au dehors et le manque de lumière permet l'accès à l'espace du dedans plus lointain. Le sombre relève davantage du distant et du spéculatif, contrairement au lumineux qui convient au proche et au narratif, s'il n'aveugle pas par l'excès. Dans l'ombre, l'observateur est régi par un syntagme de manipulation esthétique qui le conduit, par les résonances de son être, jusqu'à une perception aiguisée au-delà du seuil de sensibilité ordinairement soumis aux seules évidences. Cette plus-value perceptive et pathémique, en raison des axiologies à l'œuvre, débouche sur une plus-value modale qui constitue un nouveau mode d'accès à la connaissance. La valeur qui se construit est celle d'une profondeur esthétique exclusive, fondée sur la lenteur en raison de la clôture de la saisie perceptive de l'objet et sur l'investissement du sujet percevant qui l'habille à sa guise.

À ces procédés énonciatifs conjugués, «méréologique» et chromatique, indissociables des configurations de lumière, correspond une épistémologie spécifique qui a pu heurter, à son insu, le commanditaire originel. S'il est impossible à l'énonciateur du XVI $\mathrm{X}^{\mathrm{e}}$ siècle de ne pas traiter le récit biblique, et ceci d'autant plus qu'il effectue une œuvre de commande, il opère un découpage inhabituel dans le récit canonique et, par cette manipulation, il en modifie le contenu. C'est aussi pourquoi nous pouvons parler d'une esthétique passionnelle, où la véritable dramatisation ne dépend pas du programme narratif identifié, mais bien plus de l'énonciation soumise au rôle actantiel de la lumière qui le perturbe.

L'accomplissement du récit canonique n'est pas compromis, mais stoppé dans le parcours narratif avant la phase de transformation du sujet énoncif de l'humain en divin: quand la femme devient Sainte. Le sujet observateur est ramené essentiellement à luimême devant cet énoncé, qui se soustrait à sa fonction didactique puisque le tableau devait être exposé dans un site religieux. Si le titre guide l'observateur vers le paradigme biblique ${ }^{5}$, tous les procédés d'énonciation se conjuguent pour accorder à cette mort une interprétation à caractère essentiellement humain, qui s'oppose à la fonction d'une ouvre d'art dans une église: faire-voir pour faire-croire. Le récit de l'Assomption de la Vierge est en perspective mais reste second; à la Dormition canonique et euphémique se substitue une véritable mort dans un espace obscurci, où l'ombre s'est imposée et, avec elle, la perception d'un mystère dans le parcours des simulacres existentiels que chaque sujet s'approprie à sa guise. Mais plus la densité des ombres est forte, moins on perçoit les couleurs et les objets, et plus on construit de simulacres.

Dans le champ de présence que nous avons décrit, les variations de lumière rompent avec les stéréotypes vite saisis, voire plaqués sur le perçu pour l'occulter. L'observateur peut se livrer à une réflexion sur le visible, sur "ce que je vois vraiment", dirait MerleauPonty, et, par voie de conséquence, sur sa propre ontologie. Voilà ce qui se joue dans la présence, l'espace est rendu au temps, le temps de la méditation est devenu visible et l'énonciateur invite à le partager. Le titre d'un énoncé visuel comme La Mort de la Vierge induisait une attente en termes iconiques et plastiques avec un programme narratif relevant du divin. Or le discours nous renvoie vers l'humain. La coexistence des deux catégories est concevable, mais dans une hiérarchie inversée. Même si le discours biblique mêle les deux catégories en un Dieu fait Homme ou en une Femme Mère de Dieu, la représentation canonique des séquences bibliques pondère cette coexistence catégorielle en faveur du divin. C'est le cas dans le tableau de Saraceni, connu sous le même titre, qu'on lui préféra, et qui présente une vision idéalisée, plus convenue à cette époque. Chez Le Caravage, les diverses strates de l'énonciation réalisent un énoncé marqué par la corporéité, où ce qui compte, au-delà de l'iconique, c'est la façon dont la lumière opère plus que la symbolique de sa source. Elles soustraient le monde représenté à «l'équation métaphysique établie par Ficin entre la clarté et la proximité de Dieu, la luce par opposition à l'ombra» (Spear, 2000: 121), équation inspirée de saint Augustin et animée par l'idée d'un 
lien entre grâce plastique et grâce théologique. Le Caravage laïcise l'image par cette intimité avec le corporel et le terrestre ${ }^{6}$.

Nos analyses ont voulu mettre en exergue le rôle de la lumière dans une appréciation plus précise de ce qui, à notre avis, domine: les points de vue métaphysiques confrontés à l'inéluctable de la condition humaine. Il apparaît que, dans ce tableau, le travail de la lumière et de son contraire valorise l'univers des sensations. Il interroge le mythe biblique pour le mettre à l'épreuve, pour y introduire une substance strictement humaine pour dire une autre réalité, et non pour créer l'illusion baroque d'un idéal calqué sur des stéréotypes afin d'être acceptée par le conformisme d'un commanditaire.

\section{CONCLUSION}

Il apparaît clair que la dimension plastique du représenté signifie plus que l'iconique ou le narratif seuls. Plus l'ombre s'étend, plus elle devient la source de vérités substantielles - paradoxe qui rejoint le mythe grec du devin aveugle ou celui d'un CEdipe qui se crève les yeux pour mieux voir dans l'obscurité totale. On comprend ainsi le paradoxe qui fait que, d'une part, les modalités de la perception sont à l'origine de l'expérience esthésique et d'une philosophie de l'existence alimentées par l'imagination des profondeurs, et que, d'autre part, l'énigme de la perception reste liée à l'aptitude du sujet à la présence.

L'important ici est l'efficience des procédés, particulièrement dans l'utilisation de la lumière, pour cette esthétique simultanément figurative et figurale, qui contraint l'observateur à se confronter à ses propres modalités de connaissance et de croyance face à ce qui n'est ni intelligible, ni sensible, mais d'un autre ordre. Au bout du compte, la mise en lumière a pour seul objectif de donner à penser cet invisible grâce à un discours qui porte le destinataire à la hauteur d'une métaphysique affranchie des contraintes dogmatiques, peut-être pour une éthique attachée au monde sensible et focalisée sur le corps par la lumière.

\section{N O TES}

1. Le caravagisme à sa suite - même si la notion est contestable - sera marqué par une terminologie révélatrice de la part de la lumière dans l'analyse de cette production que l'histoire de l'art segmente en diurnes et nocturnes. Les nocturnes sont éclairés par une source lumineuse artificielle, présente dans le tableau, contrairement aux diurnes qui figurent une lumière solaire. Historiquement, dans le nocturne, la nuit n'est plus représentée par un fond bleu étoilé mais, dans ses effets, par des contrastes accusés d'ombres et de lumières et par un obscurcissement général des tons.

2. Voir J. de Voragine (1967 : 86-111), «L'Assomption de la bienheureuse Vierge Marie». Ce passage offre un bref récit de la mort de la Vierge: "Quand ceux qui dormaient furent éveillés, continue saint Cosme, et qu'ils virent sans vie le corps de la Vierge, ils se livrèrent à une grande tristesse et poussèrent des gémissements \%. La brièveté de l'épisode contraste avec la pléthore de détails concernant son Assomption. Le texte tisse alors l'isotopie de la lumière "immense" en permanence associée aux sons (chœurs, voix, louanges, cantiques, hymnes, instruments de musique des troupes angéliques) et aux manifestations passionnelles euphoriques. Le récit de saint Jean, dans les textes apocryphes, y associe le parfum mais ne rend pas compte de

l'instant précis de la mort (F. Bovon et P. Geoltrain, 1997: 183-188).

3. Nous attirons l'attention sur les pages 147-152 consacrées, dans cet ouvrage, à la description minutieuse de l'émergence des couleurs dans ce tableau.

4. N. Roelens, «La bouche ouverte en peinture comme objet sémiotique: du cri au geste ", dans F. Parouty-David et C. Zilberberg, 2003.

5. La mort de la Vierge est habituellement considérée comme un court sommeil, d'où elle fut miraculeusement enlevée par les anges pour être conduite au Ciel. Elle n'a donc pas de caractère dramatique. Elle ne ressemble pas à une mort d'être humain.

6. On peut penser que cette analyse complète celle qui prévalut au XVII ${ }^{\mathrm{e}}$ siècle et qui fit refuser cette toile dans le site pour lequel elle avait été commandée. Réalisée pour la chapelle de Santa Maria della Scala de Trastevere à Rome, elle fut jugée indigne des lieux sur un motif assez réducteur.

\section{RÉfÉREN CES BIBLIO g RAPH IQ U ES}

ALEKAN, H. [2001]: Des lumières et des ombres, Paris, Éd. du collectionneur, 12-14.

BANU [2000]: L'Homme de dos. Peinture, théâtre, Paris, Adam Biro. BARDON, F. [1997]: Caravage ou l'expérience de la matière, Paris, P.U.F. BOVON, F. et P. GEOLTRAIN (sous la dir. de) [1997]: Écrits apocryphes chrétiens, Paris, Gallimard.

Deleuze, G. [(1988) 1997]: Le Pli. Leibniz et le baroque, Paris, Éd. de Minuit, coll. "Critique».

FONTANILLE, J. [1995]: Sémiotique du visible, Paris, P.U.F.;

[1989]: Les Espaces subjectifs. Introduction à la sémiotique de l'observateur, Paris, Hachette.

Greimas, A.J. [1987]: De l'imperfection, Périgueux, Éd. Fanlac. Hegel [(1832) 2002]: Leçons d'esthétique, III e partie, 3e section, chap. 1 ("Caractère général de la peinture»), Paris, Hatier.

MERLEAU-PONTY, M. [(1979) 2002]: Le Visible et l'Invisible suivi de Notes de travail, Paris, Gallimard, coll. «Tel».

PAROUTY-DAVID, F. et C. ZilberberG (sous la dir. de) [2003]:

Sémiotique et Esthétique, Limoges, PULIM.

SPEAR, R. [2000]: "Caravage et La Tour: ténèbres et lumières de la grâce ", L'Âge d'or du nocturne, Paris, Gallimard, coll. «art et artistes ". Voragine, J. de [1967]: La Légende dorée, Paris, Garnier Flammarion. 